\title{
3D Near-field Source Localization for Cross Array via Fourth-order Cumulant
}

\section{Zhong-Xi XIA ${ }^{1, a,{ }^{*}, \text {, Hao-Yu YANG }}{ }^{1, b}$, Wei-Tao LIU ${ }^{1, c}$, Qian-Lin CHENG ${ }^{1, d}$ and Xiao-Fei ZHANG ${ }^{1, \mathrm{e}}$}

\author{
${ }^{1}$ College of Electronic and Information Engineering, Nanjing University of Aeronautics and \\ Astronautics
}

axiazhongxi@nuaa.edu.cn, byy13794yy@163.com,cliuweitao@nuaa.edu.cn, dchengqianlin_nuaa@163.com, 'zhangxiaofei@nuaa.edu.cn

${ }^{*}$ Corresponding author

Keywords: Near-field, 3D localization, Cross array, Fourth-order cumulant.

\begin{abstract}
This paper proposes a algorithm for 3D near-field source localization, which uses the cross array and fourth-order cumulant. We construct two high-dimensional matrices by using these six fourth-order cumulant matrices, according to the subspace theory, we can extract the rotation factors, which contain the parameter information of the sources. Then it is possible to obtain the elevation angle, azimuth angle and range of the sources. This algorithm doesn't need spectral peak search.The high degree of freedom of the higher order cumulants makes it unique advantages over traditional methods, such as the ability to avoid loss of apertures and the natural resistance to Gaussian noise. The results of MATLAB simulation show that this is an effective three-dimensional parameter estimation algorithm for near-field source.
\end{abstract}

\section{Introduction}

Source localization can be classified into far-field source localization and near-field source localization according to the range between the sources and the array. In both cases, the wave-fronts of incoming signals are different completely, so that they have different signal models $[1,2]$. In the far-field source localization [3-6], the wave-front of the incoming signal is assumed to be a plane wave propagating in space, so each source is parameterized by only the Direction-Of-Arrival (DOA).But in the near-field, the signal wave-front is spherical, and both the DOAs and ranges are needed [7-17]. The algorithms in reference [18-20] were addressed to deal with three-dimensional (3D) source localization, which are joint azimuth angle, elevation angle and range estimation problem. But if they need spectral peak search that will lead compute very complexly.

In reference [1], it defined fourth-order cumulant matrix from the received signal, then constructed a high-dimensional matrix, and used the subspace theory to obtain the parameter estimation of the angle and range of the source, but this method can only get two-dimensional parameter estimation. We learned and used this method, so proposed a method for 3D near-field source location using cross-array and fourth-order cumulant. So that we can get three-dimensional parameter estimation. A cross-array which in the XOY plane is proposed in reference [2], while the Y axis has only three elements. It can get the three-dimensional parameter estimation of the source via the fourth-order cumulant, but the utilization efficiency of the element is relatively low. Compared the two methods, the algorithm in reference [2] suffers a heavy loss of the array aperture. The algorithm in this paper has a good estimation accuracy and performance, avoiding the aperture loss, which has a great impact on the resolution. Also, the high degree of freedom of the higher order cumulant makes it unique advantages over traditional methods, such as it does not require spectral peak search, the ability to avoid loss of apertures and the natural resistance to Gaussian noise. The results of MATLAB simulation show that this is an effective three-dimensional parameter estimation method for near-field source, its performance is better. 


\section{Data Model}

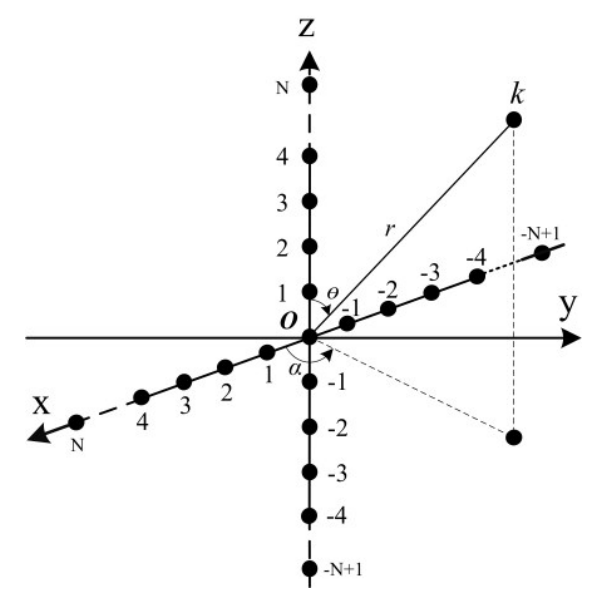

Fig. 1. Cross-array for 3D near-field source localization

Similar to [1, 2], we consider that there are K narrowband and independent non-Gaussian near-field radiation sources radiated to the cross array, as shown in Fig. 1. Each subarray contains $2 \mathrm{~N}$ equally spaced omnidirectional sensors, the interval between two adjacent elements is $\lambda / 4$, and the intersection of the array is selected as the reference point of the phase. After being down-converted to baseband and sampled at a proper sampling rate that satisfies the Nyquist rate, the signals received by the $(i, 0)$-th and $(0, i)$-th sensors in the subarray can be expressed as follows,

$$
z_{i}(t)=\sum_{k=1}^{K} S_{k}(t) \cdot e^{j\left(\gamma_{z k} i+\phi_{z k} i^{2}\right)}+n_{z i}(t)
$$

where $\gamma_{z k}=-2 \pi \frac{d}{\lambda} \cos \theta_{k}, \phi_{z k}=\pi \frac{d^{2}}{\lambda_{k} r_{k}} \sin ^{2} \theta_{k},(1 \leq k \leq K)$

$$
x_{i}(t)=\sum_{k=1}^{K} S_{k}(t) \cdot e^{j\left(\gamma_{x k} i+\phi_{x i k}{ }^{2}\right)}+n_{x i}(t)
$$

where $\gamma_{x k}=-2 \pi \frac{d}{\lambda_{k}} \sin \theta_{k} \cos \alpha_{k}, \phi_{x k}=\pi \frac{d^{2}}{\lambda_{k} r_{k}}\left(1-\sin ^{2} \theta_{k} \cos ^{2} \alpha_{k}\right) \quad(1 \leq k \leq K)$.

while $n_{z i}(t)$ and $n_{x i}(t)$ represent the additive measurement noise, $\theta_{k}, \alpha_{k}$ and $r_{k}$ denote the elevation angle, azimuth angle and range of the $k$-th source, for $k=1, \ldots, K$, respectively.

The same, similar to the reference [1], throughout the rest of the paper, the following hypotheses are assumed to hold.

(1) The sources are statistically mutually independent of non-Gaussian narrow-band stationary processes, with non-zero kurtosis.

(2) The sensor noise is zero-mean Gaussian signal and independent of the source signals, which variance is $\sigma^{2}$.

(3) The source parameters are different from each other, that is $\phi_{x i} \neq \phi_{x j}, r_{i} \neq r_{j}$.

(4) The distance between the elements must be met $d \leq \min \left(\lambda_{k} / 4\right)$, the number of sources and the number of elements must be met $K<2 N-2$. 


\section{Algorithm Description}

\subsection{Define Six Fourth-order Cumulant Matrices}

Similar to [1], we defined the fourth-order cumulant matrix $C_{1}$, for different sensor and time lags, the $(m, n)$-th element of which has the following form,

$$
C_{1}(m, n)=\operatorname{cum}\left\{z_{m-N+1}(t), z_{m-N}^{*}(t), z_{n-N+1}^{*}(t), z_{n-N}(t)\right\}=\sum_{k=1}^{K} c_{4 s k} e^{j 2(m-n) \phi_{z k}},
$$

where $1 \leq m, n \leq 2 * N-2, c_{4 s k}=\operatorname{cum}\left\{s_{k}(\mathrm{t}), s^{*}{ }_{k}(\mathrm{t}), s^{*}{ }_{k}(\mathrm{t}), s_{k}(\mathrm{t})\right\}$ is the the fourth-order kurtosis of the $k$-th source. Note that $C_{1}$ can be represented in a compact form as

$$
C_{1}=A_{z} C_{4 s} A_{z}^{H}
$$

The superscript $H$ denotes the Hermitian transpose, $C_{4 s}=\operatorname{diag}\left[c_{4 s 1}, \ldots, c_{4 s K}\right], A_{z}=\left[a_{z 1}, \ldots, a_{z K}\right]$. Likewise, the following can be obtained,

$$
\begin{gathered}
C_{2}(m, n)=\operatorname{cum}\left\{z_{(m+1)-N+1}(t), z_{(m+1)-N}^{*}(t), z_{n-N+1}^{*}(t), z_{n-N}(t)\right\}=\sum_{k=1}^{K} c_{4 s k} e^{j 2 \phi_{z k}} e^{j 2(m-n) \phi_{z k}}=A_{z} \Phi_{z 1} C_{4 s} A_{z}^{H}, \\
C_{3}(m, n)=\operatorname{cum}\left\{z_{m-N+1}(t), z_{m-N}^{*}(t), z_{n-N+1}^{*}(t), z_{n-N}(t)\right\}=\sum_{k=1}^{K} c_{4 s k} e^{j 2 \gamma_{z k}} e^{j 2(m-n) \phi_{z k}}=A_{z} \Phi_{z 2} C_{4 s} A_{z}^{H},
\end{gathered}
$$

where $\Phi_{z 1}=\operatorname{diag}\left\{e^{j 2 \phi_{z 1}}, e^{j 2 \phi_{z 2}}, \ldots, e^{j 2 \phi_{z K}}\right\}, \Phi_{z 2}=\operatorname{diag}\left\{e^{j 2 \gamma_{z 1}}, e^{j 2 \gamma_{z 2}}, \ldots, e^{j 2 \gamma_{z K}}\right\}, 1 \leq m, n \leq 2 N-2$.

Also the following can be get,

$$
\begin{gathered}
C_{4}(m, n)=\operatorname{cum}\left\{x_{m-N+1}(t), x_{m-N}^{*}(t), x_{n-N+1}^{*}(t), x_{n-N}(t)\right\}=\sum_{k=1}^{K} c_{4 s k} e^{j 2(m-n) \phi_{x k}}=A_{x} C_{4 s} A_{x}^{H}, \\
C_{5}(m, n)=\operatorname{cum}\left\{x_{(m+1)-N+1}(t), x_{(m+1)-N}^{*}(t), x_{n-N+1}^{*}(t), x_{n-N}(t)\right\}=\sum_{k=1}^{K} c_{4 s k} e^{j 2 \phi_{x k}} e^{j 2(m-n) \phi_{x k}}=A_{x} \Phi_{x 1} C_{4 s} A_{x}^{H}, \\
C_{6}(m, n)=\operatorname{cum}\left\{x_{m-N+1}(t), x_{m-N}^{*}(t), x_{n-N+1}^{*}(t), x_{n-N}(t)\right\}=\sum_{k=1}^{K} c_{4 s k} e^{j 2 \gamma_{x k}} e^{j 2(m-n) \phi_{x k}}=A_{x} \Phi_{x 2} C_{4 s} A_{x}^{H},
\end{gathered}
$$

where $A_{x}=\left[a_{x 1}, a_{x 2}, \ldots, a_{x K}\right], \Phi_{x 1}=\operatorname{diag}\left\{e^{j 2 \phi_{x 1}}, e^{j 2 \phi_{x 2}}, \ldots, e^{j 2 \phi_{x K}}\right\}, \Phi_{x 2}=\operatorname{diag}\left\{e^{j 2 \gamma_{x 1}}, e^{j 2 \gamma_{x 2}}, \ldots, e^{j 2 \gamma_{x K}}\right\}$, $1 \leq m, n \leq 2 N-2$. Since all the source signals are assumed to have nonzero kurtosis, $C_{4 s}$ is an invertible diagonal matrix. Besides, because of the assumption $i \neq j, \phi_{x i} \neq \phi_{x j}, \gamma_{z i} \neq \gamma_{z j}$ and $r_{i} \neq r_{j}$, So $A_{z}$ and $A_{x}$ are all $(2 N-2) \times(2 N-2)$ Vandermonde matrices with full column rank $K, C_{1} \sim C_{6}$ are all $(2 N-2) \times(2 N-2)$ dimensional matrices with rank $K$.

We can construct a high-dimensional matrix,

$$
C_{z}=\left[C_{1} C_{2} C_{3}\right]^{T}=\left[\begin{array}{lll}
A_{z} C_{4 s} A_{z}^{H} & A_{z} \Phi_{z 1} C_{4 s} A_{z}^{H} A_{z} \Phi_{z 2} C_{4 s} A_{z}^{H}
\end{array}\right]^{T}=\left[\begin{array}{lll}
A_{z} & A_{z} \Phi_{z 1} A_{z} \Phi_{z 2}
\end{array}\right]^{T} C_{4 s} A_{z}^{H}=\%_{z} C_{4 s} A_{z}^{H}
$$

The same operation, we can get $C_{x}=\left[\begin{array}{lll}C 4 & C 5 & C 6\end{array}\right]^{T}$, the superscript $T$ denotes transpose. 


\subsection{Parameter Estimation}

Singular value decomposition to the high-dimensional matrices, for example

$$
C_{z}=U_{z} S_{z} V_{z}^{H}
$$

While $U_{z}, S_{z}$ are $(6 N-6) \times(6 N-6)$ high-dimensional matrices, and $V_{z}$ is $(2 N-2) \times(2 N-2)$ dimensional matrix, Let the left singular value vector matrix $U_{z}=\left[u_{z 1}, u_{z 2}, \ldots, u_{z(6 N-6)}\right]$, and the singular value vectors corresponding to $K$ larger singular values are taken out, so we can get $E_{z}=\left[u_{z 1}, u_{z 2}, \ldots, u_{z k}\right]$. From the subspace theory $E_{z} T=\mathscr{A}_{z}^{0}, E_{z}$ could be divided into three $(2 N-2) \times K$ dimensional matrices $E_{z a}, E_{z b}, E_{z c}$, So that we can get,

$$
\left[\begin{array}{lll}
E_{z a} & E_{z b} & E_{z c}
\end{array}\right]^{T} T=E_{z} T=A_{z}^{0}=\left[\begin{array}{lll}
A_{z} & A_{z} \Phi_{z 1} & A_{x} \Phi_{x 2}
\end{array}\right]^{T}
$$

From the formula (14), we can get two important equations, $E_{z a}^{+} E_{z b}=T^{-1} \Phi_{z 1} T, E_{z a}^{+} E_{z c}=T^{-1} \Phi_{z 2} T$. In the above formula, the " $(\cdot)^{+}$" denotes pseudo inverse, Extract the rotation factors and perform the eigenvalue decomposition operation. It can be found that the extraction of the rotation factor to get the eigenvalue can obtain the parameter information of the source, the parameter $\gamma_{z k}$ and $\phi_{z k}$ which containing the location information of the sources can be obtained from $\Phi_{z 1}, \Phi_{z 2}$, $\hat{\gamma}_{z k}=\angle \cdot\left(\Phi_{z 2}\left(k^{\prime}\right)\right) / 2, \hat{\phi}_{z k}=\angle \cdot\left(\Phi_{z 1}\left(k^{\prime}\right)\right) / 2$, the " $\angle \cdot$ " denotes the phase angle operator. From these intermediate variables, we can get the multidimensional parameter estimation of the source

$$
\begin{aligned}
& \hat{\theta}_{k}=\arcsin \left(-\hat{\gamma}_{x k} \lambda / 2 \pi d\right), \\
& \hat{r}_{z k}=\left[\pi d^{2} \cos ^{2}\left(\hat{\theta}_{k}\right)\right] / \lambda_{k} \hat{\phi}_{z k} .
\end{aligned}
$$

The same operation is used for $C_{x}$, so we can get the parameters estimation in another dimension, that is, $\hat{\alpha}_{k}=\arcsin \left(-\hat{\gamma}_{z k} \lambda_{k} / 2 \pi d\right), \hat{r}_{x k}=\left[\pi d^{2} \cos ^{2}\left(\hat{\alpha}_{k}\right)\right] / \lambda_{k} \hat{\phi}_{x k}$

\subsection{Parameters Pairing}

Similar to the reference [1], find the position of the largest element of the modulus in each row of $R_{t t}$, we can get the correct order of elevation angle and range parameters from the source.

$$
R_{t t}=\operatorname{abs}\left(T_{1}^{H}(:, \mathrm{i}) * T_{2}(:, \mathrm{j})\right)\left\{\begin{array}{l}
\approx 1, \text { Corresponds to the same eigenvector } \\
\approx 0, \text { Corresponds to the different eigenvector }
\end{array}\right.
$$

Also we can get azimuth angle and the correct corresponding range parameters again. It can be seen in the hypothesis, the range from different sources are different, $r_{i} \neq r_{j},[1,2]$, compare the results of the range parameter estimates obtained twice to find the correspondence.

$$
k^{\prime}=\underset{1 \leq k^{\prime} \leq K}{\arg \min }\left|\hat{r}_{x k}-\hat{r}_{z k}\right| \quad\left(1 \leq k^{\prime} \leq K\right)
$$

\subsection{Algorithm steps}

The proposed method can be described as follows.

Step1. Estimate fourth-order cumulant matrices $\hat{C}_{1}, \hat{C}_{2}, \hat{C}_{3}, \hat{C}_{4}, \hat{C}_{5}$ and $\hat{C}_{6}$, then construct $C_{z}$ and $C_{x}$. 
Step2. Singular value decomposition to the high-dimensional matrices $C_{z}$ and $C_{x}$, then according to the subspace theory, get the rotation factors $\Phi_{z 1}, \Phi_{z 2}, \Phi_{x 1}, \Phi_{x 2}$, Eigenvalue decomposition of these rotation factors., get $\hat{\gamma}_{z k}, \hat{\phi}_{z k}$ and $\hat{\gamma}_{x k}, \hat{\phi}_{x k}$ which containing the location information of the sources.

Step3. Match the parameters $\hat{\gamma}_{z k}$ and $\hat{\phi}_{z k}, \hat{\gamma}_{x k}$ and $\hat{\phi}_{x k}$, get the parameters estimation $\left(\hat{\theta}, \hat{r}_{z}\right)$ and $\left(\hat{\alpha}, \hat{r}_{x}\right)$ respectively.

Step4. Match the two sets of data $\left(\hat{\theta}, \hat{r}_{z}\right)$ and $\left(\hat{\alpha}, \hat{r}_{x}\right)$, get three-dimensional parameter estimation of the source $(\hat{\theta}, \hat{\alpha}, \hat{r})$.

\section{Simulation Results}

Assumptions are as described above, consider there are 2 near-field sources located at $\left\{\theta_{1}=40^{\circ}, \alpha_{1}=50^{\circ}, r_{1}=0.9 \lambda\right\}$ and $\left\{\theta_{2}=50^{\circ}, \alpha_{2}=45^{\circ}, r_{2}=0.3 \lambda\right\}$, Each Monte Carlo simulation experiment runs 500 times independently. The performance of the algorithm is measured by the root mean square error (RMSE) of the estimated parameters, for example,

$$
R M S E_{\theta}=\sqrt{\frac{1}{M} \sum_{m=1}^{M}\left(\hat{\theta}_{m}-\theta\right)^{2}} .
$$

Fig. 2, Fig. 3 and Fig. 4 depict the RMSE of the parameter estimation when the number of snapshots is 300, 500 and 700, respectively. Fig. 5, Fig. 6 and Fig. 7 depics the scatter figure of angle and range in different dimension, respectively. Fig. 8, Fig. 9 and Fig. 10 compares the proposed algorithm and the algorithm in reference [2], respectively, while the number is set equal to 500, and the SNR varies from $5 \mathrm{db}$ to $25 \mathrm{db}$. Fig. 10, Fig. 11 and Fig. 12 compares the proposed algorithm and the algorithm in reference [2], respectively, while the SNR is set equal to $20 \mathrm{db}$, and the snapshot number varies from 20 to 1000 .

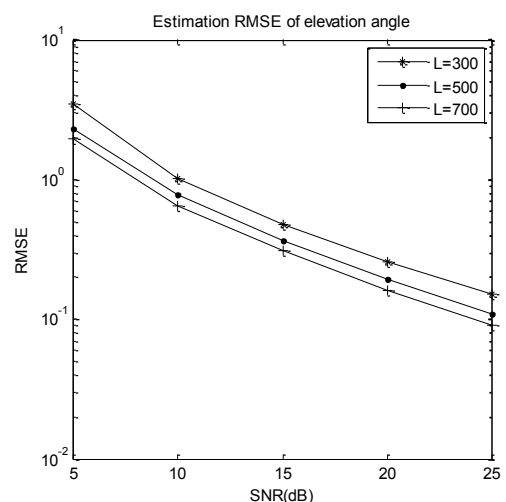

Fig. 2. Estimation RMSE of angle

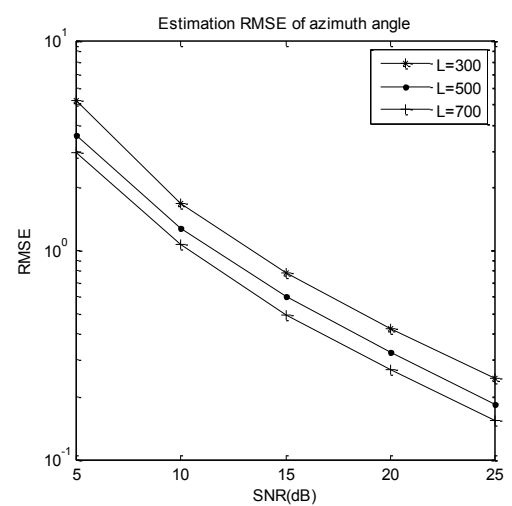

Fig. 3. Estimation RMSE of azimuth angle

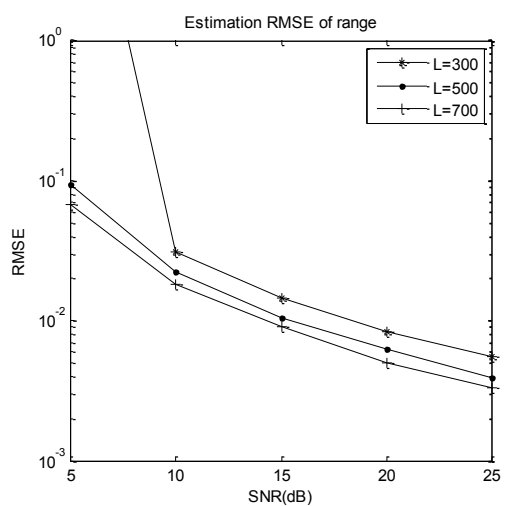

Fig. 4. Estimation RMSE elevation range 


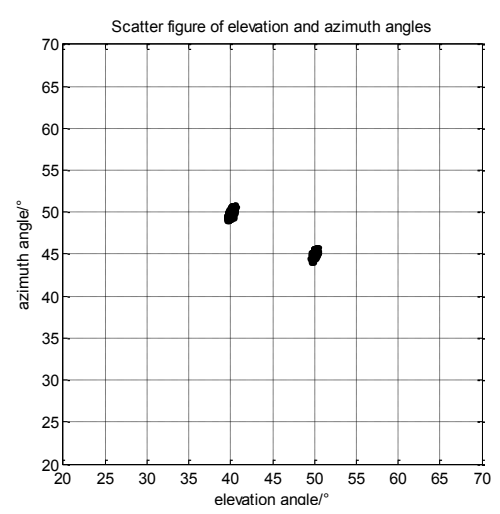

Fig. 5. Scatter figure of elevation and azimuth angles

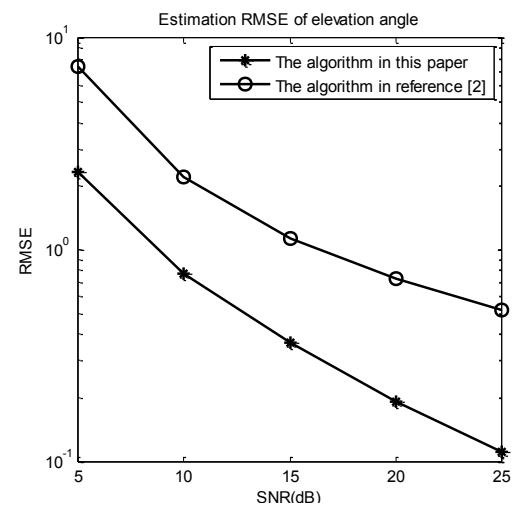

Fig. 8. Estimation RMSE of elevation angle

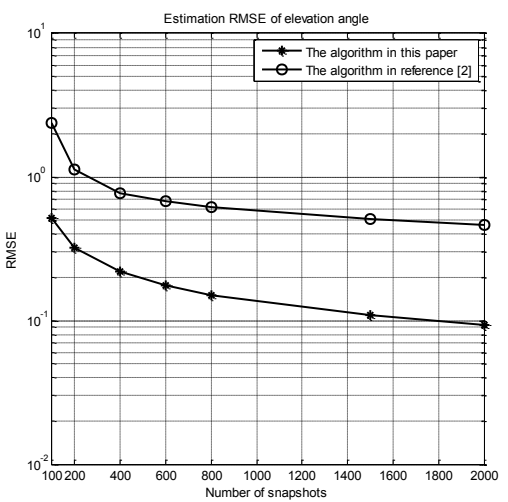

Fig. 11. Estimation RMSE of elevation angle

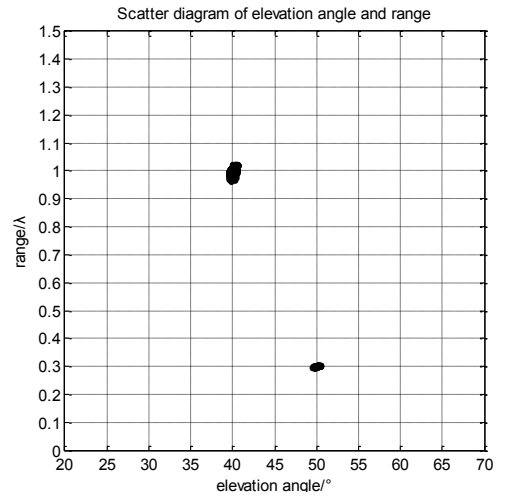

Fig. 6. Scatter figure of elevation angle and range

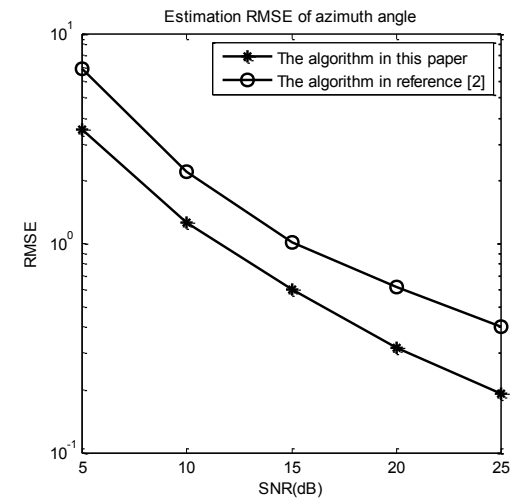

Fig. 9. Estimation RMSE of azimuth angle

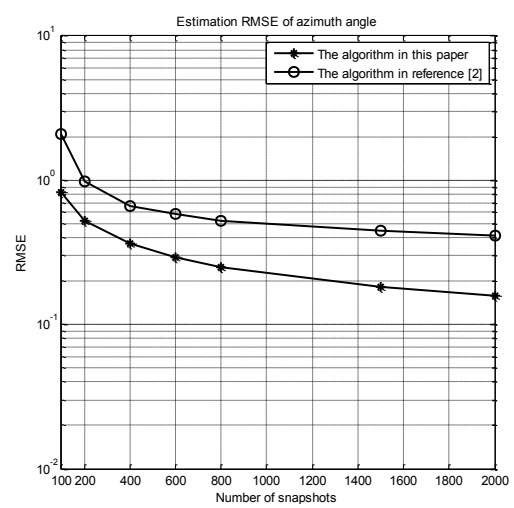

Fig. 12. Estimation RMSE of azimuth angle

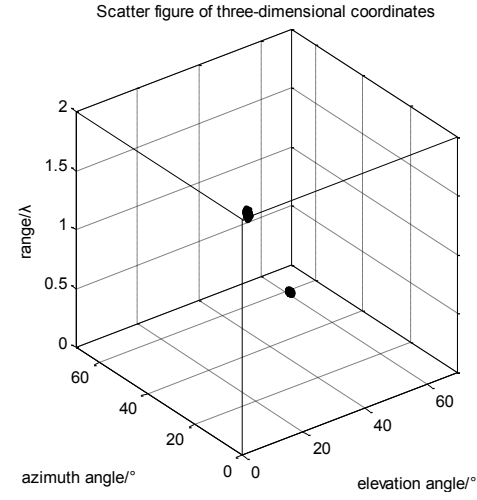

Fig. 7. Three-dimensional coordinates

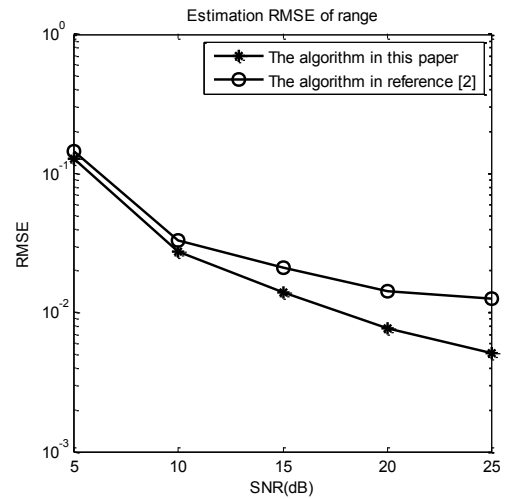

Fig. 10. Estimation RMSE of angle

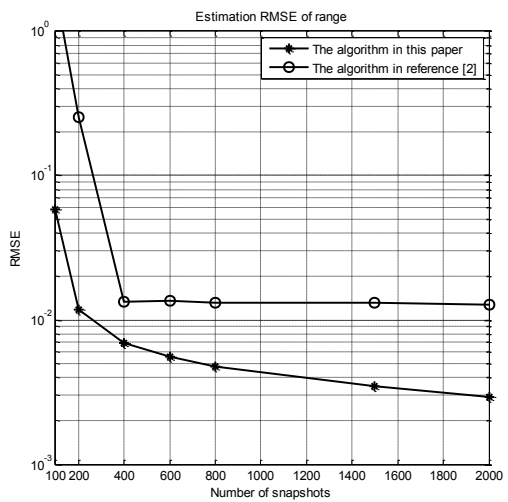

Fig. 13. Estimation RMSE of range

\section{Conclusion}

We propose a cross array in the $X O Z$ plane, construct six fourth-order cumulant matrices, which can construct two high-dimensional matrices. According to the spatial theory, we can extract the rotation factor to obtain the multidimensional parameter estimation of the source, but in the result, we must do the parameter matching work. Theoretical analysis and computer simulation results show that the algorithm is effective. 


\section{References}

[1] Junli Liang, Bangjie Ji, Feng Zhao, Junying Zhang,New parameter estimation algorithm for near-field sources. JOURNAL OF XIDIAN UNIVERSITY.Oct.2007.Vol.34 No.5R

[2] Bo Wang, Shuxun Wang, Xiao Han. New method for three dimensional parameters estimation of near field sources. Journal of Jilin University Vol.34 No.4 Oct.2004

[3] S. M. Kay, Fundamentals of Statistical Signal Processing: Estimation Theory, Prentice-Hall, Upper Saddle River, NJ, USA, 1993.

[4] R. O. Schmidt, "Multiple emitter location and signal parameter estimation," IEEE Transactions on Antennas and Propagation, vol. 34, no. 3, pp. 276-280, 1986.

[5] R. Roy and T. Kailath, "ESPRIT - estimation of signal parameters via rotational invariance techniques," IEEE Transactions on Acoustics, Speech, and Signal Processing, vol. 37, no. 7, pp.984-995, 1989.

[6] H. Krim and M. Viberg, "Two decades of array signal processing research: the parametric approach,” IEEE Signal Processing Magazine, vol. 13, no. 4, pp. 67-94, 1996.

[7] A. L. Swindlehurst and T. Kailath, "Passive direction-of-arrival and range estimation for near-field sources," in Proceedings of the 4th Annual ASSP Workshop on Spectrum Estimation and Modeling, pp. 123-128, Minneapolis, Minn, USA, August 1988.

[8] Y.-D. Huang and M. Barkat, "Near-field multiple source localization by passive sensor array," IEEE Transactions on Antennas and Propagation, vol. 39, no. 7, pp. 968-975, 1991.

[9] R. Jeffers, K. L. Bell, and H. L. Van Trees, "Broadband passive range estimation using MUSIC," in Proceedings of IEEE International Conference on Acoustics, Speech, and Signal Processing (ICASSP '02), vol. 3, pp. 2921-2924, Orlando, Fla, USA, May 2002.

[10] A. J.Weiss and B. Friedlander,"Range and bearing estimation using polynomial rooting," IEEE Journal of Oceanic Engineering, vol. 18, no. 2, pp. 130-137, 1993.

[11] D. Starer and A. Nehorai, "Passive localization on near-field sources by path following," IEEE Transactions on Signal Processing, vol. 42, no. 3, pp. 677-680, 1994.

[12] E. Grosicki, K. Abed-Meraim, and Y. Hua, "A weighted linear prediction method for near-field source localization," IEEE Transactions on Signal Processing, vol. 53, no. 10, part 1, pp.3651-3660, 2005.

[13] K. Abed-Meraim, Y. Hua, and A. Belouchrani, "Second-order near-field source localization: algorithm and performance analysis," in Proceedings of the 30th Asilomar Conference on Signals, Systems, and Computers, vol. 1, pp. 723-727, Pacific Grove, Calif, USA, November 1996.

[14] R. N. Challa and S. Shamsunder, "High-order subspace based algorithms for passive localization of near-field sources," in Proceedings of the 29th Asilomar Conference on Signals, Systems, and Computers, vol. 2, pp. 777-781, Pacific Grove, Calif, USA,October 1995.

[15] N. Yuen and B. Friedlander, "Performance analysis of higher order ESPRIT for localization of near-field sources,” IEEE Transactions on Signal Processing, vol. 46, no. 3, pp. 709-719,1998.

[16] J.-F. Chen, X.-L. Zhu, and X.-D. Zhang, "A new algorithm for joint range-DOA-frequency estimation of near-field sources", EURASIP Journal on Applied Signal Processing, vol. 2004, no. 3,pp. 386-392, 2004.

[17] Y. Wu, L. Ma, C. Hou, G. Zhang, and J. Li, "Subspace-based method for joint range and DOA estimation of multiple nearfield sources," Signal Processing, vol. 86, no. 8, pp. 2129-2133,2006.

[18] N. Kabaoglu, H. A. Cirpan, E. Cekli, and S. Paker, "Maximum likelihood 3-D near-field source localization using the EM algorithm" in Proceedings of the 8th IEEE International Sympo- 
sium on Computers and Communication (ISCC '03), vol. 1, pp.492-497, Kiris-Kemer, Turkey, June-July 2003.

[19] H.-S. Hung, S.-H. Chang, and C.-H. Wu, "3-D MUSIC with polynomial rooting for near-field source localization," in Proceedings of IEEE International Conference on Acoustics, Speech, and Signal Processing (ICASSP '96), vol. 6, pp. 3065-3068, Atlanta, Ga, USA, May 1996.

[20] K. Abed-Meraim and Y. Hua, "3-D near field source localization using second order statistics," in Proceedings of the 31st Asilomar Conference on Signals, Systems, and Computers, vol. 2, pp. 1307-1311, Pacific Grove, Calif, USA, November 1997. 